\title{
Autonomous sensory meridian response: Your patients already know, do you?
}

\section{ABSTRACT}

Public interest in autonomous sensory meridian response (ASMR) is growing on digital media platforms. Some people can elicit the response by watching videos containing triggering sounds and images. People susceptible to ASMR's effects report tingling sensations on the head and neck, as well as feelings of euphoria, relaxation, and mood elevation. Underlying mechanisms of the phenomenon are not well understood, but physiologic evidence corroborates some of the self-reported positive effects. Healthcare professionals should be aware of this emerging topic, and the potential for therapeutic applications should be investigated.

\section{KEY POINTS}

ASMR involves pleasurable feelings resulting from audiovisual stimuli, such as tapping sounds, watching someone brush their hair, or having something explained in detail in a whisper.

Differences between people capable of the ASMR response and controls have been detected in personality traits, neural activity, and functional brain connectivity.

ASMR has been anecdotally reported to improve symptoms of anxiety, depression, insomnia, and chronic pain.

Many videos designed to elicit ASMR are freely available on YouTube and other platforms.

The authors report no relevant financial relationships which, in the context of their contributions, could be perceived as a potential conflict of interest.

doi:10.3949/ccjm.87a.20005
UTONOMOUS SENSORY MERIDIAN RESPONSE

(ASMR) is an atypical sensory-emotional phenomenon triggered in some people by specific audiovisual stimuli. It is characterized as a pleasant, tingling sensation of the head or neck, and is accompanied by positive feelings, such as relaxation, a sense of well-being, euphoria, and mood elevation. Common triggers include watching someone whispering, engaging in repetitive rhythmic motions, and exploring an object. ${ }^{1}$

Although our understanding of the mechanisms behind ASMR and its potential clinical implications are in early stages, public interest in the phenomenon is growing and people are actively seeking it out as a type of complementary therapy. As in other complementary therapies such as acupuncture and massage, patients may be ahead of the evidence-based literature in their interest and use of ASMR. Many use it to help alleviate stress or pain or promote sleep as an alternative to conventional therapies, ${ }^{1}$ and they may seek information from their healthcare professionals about its use.

This article describes ASMR and summarizes published research investigating its underlying mechanisms and effects.

\section{POPULARITY OUTSIDE OF THE MEDICAL COMMUNITY}

ASMR has had a presence in Internet culture for nearly a decade. It is becoming more commonly recognized and has recently been referenced in conventional media outlets, including Good Morning America and the Netflix 
series Follow This. Despite being relatively unstudied, it has garnered a large following on digital platforms such as Reddit and YouTube, where ASMR videos are freely available.

\section{CHARACTERISTICS OF ASMR-CAPABLE PEOPLE}

Specific personality traits have been ascribed to people who are capable of ASMR. A study evaluating the Big Five Personality Inventory of 290 ASMR-capable people and 290 matched controls determined that the capable cohort scored significantly higher on openness-to-experience and neuroticism personality scales, and significantly lower on personality scales of conscientiousness, extraversion, and agreeableness. ${ }^{2}$ A positive correlation was also found between the degree of opennessto-experience and neuroticism personality dimensions and intensity of ASMR experiences.

Another study found that ASMR-capable people scored significantly higher on the mindful attention and awareness scale and the curiosity subscale of the Toronto Mindfulness Scale than matched controls, ${ }^{3}$ suggesting that ASMR-capable people tend to be more mindMany use ASMR ful than those who do not experience ASMR. These findings suggest that certain personality to help alleviate characteristics (and likely other unstudied facstress or pain or promote sleep as an alternative to conventional therapies tors) may contribute to the ability to experience the phenomenon.

\section{WHAT ASMR IS NOT}

ASMR is sometimes confused with other sensory-emotional phenomena such as misophonia, synesthesia, and frisson. These are distinct, although sometimes overlapping, conditions.

Misophonia is an aversion to sounds such as chewing, coughing, or loud breathing. While misophonia and ASMR appear to lie on opposite ends of a spectrum, ${ }^{1}$ a study found that ASMR-capable people scored higher on the Misophonia Questionnaire than matched controls, indicating that they may be more likely to have misophonia than the general population. ${ }^{4}$

Synesthesia involves external sensory stimuli triggering the internal experience of a different sensory modality. Commonly experienced synesthetic associations include per- ceiving color in response to seeing a letter of the alphabet or sensing a taste when hearing a specific word. ${ }^{5}$ Unlike ASMR, synesthesia can be regarded as a blending of senses.

Frisson, also known as musical or aesthetic chills, is the sensation of chills down the spine when listening to peak emotional moments in music. ${ }^{6}$ This positive sensation resembles those of ASMR. Neuroimaging in a small sample of ASMR-capable participants $(n=10)$ found that ASMR and frisson follow similar neurofunctional patterns of activation in areas of reward and emotion, such as the nucleus accumbens, dorsal anterior cingulate cortex, supplementary motor area, and insula. ${ }^{7-9}$ However, people with ASMR had increased activation of the medial prefrontal cortex, an area associated with social cognition, social behaviors (eg, grooming), and self-awareness, while people with frisson had reduced activation of this area. ${ }^{7-11}$

\section{KEY FEATURES ELICIT ASMR}

Although ASMR triggers can be found in daily life, many people seek online videos designed to elicit ASMR, with the most popular amassing millions of views. ${ }^{1}$ Their focus varies widely and includes personal attention, grooming, spa treatments (eg, massage, aromatherapy, haircut, make-up application), eating, cleaning, and exploring household objects. Many videos feature specific trigger sounds, such as whispering, rustling of metallic foil, tapping fingernails, scratching, crisp sounds, keyboard typing, chewing, and lip-smacking. A category of ASMR videos is dedicated to clinical roleplaying, which may include whispered narration during simulated medical history-taking and physical examinations. ${ }^{12}$ Some simulate an "authentic" experience by using props such as penlights, stethoscopes, ophthalmoscopes, and otoscopes.

ASMR intensity depends on various characteristics of the stimuli. ${ }^{13}$ The most effective triggers are about 1 to 5 minutes long, are viewed in a pleasant environment, contain subject matter that is perceived as realistic, and involve diligent exploration of an object. Low-pitched audio triggers may be more reliable at inducing ASMR than visual stimuli. 


\section{NEUROLOGIC BASIS STUDIED}

Preliminary studies have largely focused on understanding mechanisms by which ASMR elicits positive sensations in capable individuals. Functional magnetic resonance imaging-based investigations suggest that exposure to ASMR media evokes activation in certain brain areas associated with attention, social cognition, and sensory processing. ${ }^{7,9,14}$ One study performed with ASMR-capable participants revealed significant activation of the nucleus accumbens (a reward area). ${ }^{7}$ A subsequent study compared ASMR-capable participants with controls but did not identify this effect. ${ }^{14}$ The dissimilar results could be due to differences in study methods: the former study measured brain activity during the self-reported tingling sensations and the latter measured brain activity throughout the entire ASMR media viewing session.

Other studies have found that ASMR-capable people have significantly less functional connectivity between frontal lobes and sensory-attentional areas compared with controls, suggesting that ASMR-capable people have a reduced capacity to inhibit sensory-emotional experiences. ${ }^{15,16}$

Another study found that ASMR may interfere with certain aspects of executive function, suggesting that people should not engage in ASMR before performing tasks that require focused attention. ${ }^{17}$

\section{PAIN AND MOOD IMPROVEMENTS}

Some authors have suggested that ASMR can temporarily improve symptoms of depression, ${ }^{18}$ stress, ${ }^{19,20}$ and chronic pain ${ }^{21,22}$ in a manner similar to meditation and mindfulness. ${ }^{1}$

A study conducted in ASMR-capable individuals found that $80 \%$ of participants self-reported in an online questionnaire that ASMR had a positive effect on their mood. ${ }^{1}$ Among participants who scored moderate to severe on the Beck Depression Inventory, $69 \%$ reported using ASMR to ease symptoms of depression, with many reporting mood improvement. Among respondents with chronic pain, $42 \%$ reported that ASMR improved their pain symptoms, and the reduction in pain symptoms was significant $(P<.0005)$; the pain-relieving effect was maintained for 3 hours after ASMR $(P=.014)$.
ASMR-capable study participants felt significantly more excited $(P=.048)$, more calm $(P<.001)$, less stressed $(P<.001)$, less sad $(P<.001)$, and more socially connected $(P$ $<.001)$ than non-ASMR participants after watching ASMR media, as measured by the Multi-Affect Indicator scale. ${ }^{23}$

Evidence indicates that ASMR can not only induce subjective positive emotions but also objective physiologic responses. It has been found to decrease heart rate by an average of 3.41 beats per minute $(P=.028)$ and increase skin conductance (commonly used to measure emotional arousal $)^{24}$ by an average of 0.30 microsiemens $(P=.017) .^{25}$

Although the anecdotal benefits of improved pain and mood symptoms are promising, further investigation in clinical and laboratory settings is important before ASMR can be considered a therapeutic option.

\section{PLACEBO EFFECT INVESTIGATED}

Some researchers have raised the concern that the expectation of positive effects from ASMR media could act as a placebo, causing or enhancing the response. Cash et $\mathrm{al}^{26}$ investigated this possibility by showing ASMR videos and first telling viewers that they either did or did not contain ASMR triggers. Experienced ASMR users were not only able to identify the presence of ASMR triggers, but they experienced the response only when a true trigger was present. In contrast, ASMR-naive participants were more likely to be influenced by the instructions. The authors concluded that an expectancy effect exists, which may translate to a placebo effect in ASMR-naive people but not in experienced users.

Soon after the release of this study, ${ }^{26}$ Hostler et $\mathrm{al}^{27}$ rebutted some of the conclusions, arguing that the finding that ASMR-capable people reported feeling the response only when genuine triggers were present, regardless of instructions, means that ASMR is not driven by expectancy or placebo effects but is a genuine phenomenon for those able to experience it. They also pointed out that the study was unclear about the differences between ASMR-experienced and ASMR-naive groups; because the ASMR-naive group contained people with no previous exposure to ASMR videos, at least some might have

\section{Multiple ASMR videos are available for free}


been capable of experiencing the response associated with triggers. They argued that future research should use standardized tools such as the ASMR checklist ${ }^{2}$ to better define study groups of ASMR-capable and noncapable participants.

In a related study, Keizer et $\mathrm{al}^{28}$ found that people who experience ASMR are more likely than controls to experience other illusory sensory events based on verbal suggestion. Suggestibility is thought to be a factor contributing to the placebo effect, ${ }^{29}$ and the finding that ASMR-capable people may be inherently more suggestible merits further research into the potential role of placebo.

\section{REFERENCES}

1. Barratt EL, Davis NJ. Autonomous sensory meridian response (ASMR): a flow-like mental state. PeerJ 2015; 3:e851. doi:10.7717/peerj.851

2. Fredborg B, Clark J, Smith SD. An examination of personality traits associated with autonomous sensory meridian response (ASMR). Front Psychol 2017; 8:247. doi:10.3389/fpsyg.2017.00247

3. Fredborg BK, Clark JM, Smith SD. Mindfulness and autonomous sensory meridian response (ASMR). PeerJ 2018; 6:e5414. doi: $10.7717 /$ peerj. 5414

4. Janik McErlean AB, Banissy MJ. Increased misophonia in self-reported autonomous sensory meridian response. PeerJ 2018; 6:e5351. doi: $10.7717 /$ peerj. 5351

5. Ward J. Synesthesia. Annu Rev Psychol 2013; 64:49-75. doi:10.1146/annurev-psych-113011-143840

6. Harrison L, Loui P. Thrills, chills, frissons, and skin orgasms: toward an integrative model of transcendent psychophysiological experiences in music. Front Psychol 2014; 5:790. doi:10.3389/fpsyg.2014.00790

7. Lochte BC, Guillory SA, Richard CAH, Kelley WM. An fMRI investigation of the neural correlates underlying the autonomous sensory meridian response (ASMR). Bioimpacts 2018; 8(4):295-304. doi:10.15171/bi.2018.32

8. Blood AJ, Zatorre RJ. Intensely pleasurable responses to music correlate with activity in brain regions implicated in reward and emotion. Proc Natl Acad Sci USA 2001; 98(20):11818-11823. doi:10.1073/pnas. 191355898

9. del Campo MA, Kehle TJ. Autonomous sensory meridian response (ASMR) and frisson: mindfully induced sensory phenomena that promote happiness. Int J Sch Educ Psychol 2016; 4(2):99-105. doi:10.1080/21683603.2016.1130582

10. Mitchell JP, Banaji MR, Macrae CN. The link between social cognition and self-referential thought in the medial prefrontal cortex. J Cogn Neurosci 2005; 17(8):1306-1315. doi:10.1162/0898929055002418

11. Grossmann T. The role of medial prefrontal cortex in early social cognition. Front Hum Neurosci 2013; 7:340. doi:10.3389/fnhum.2013.00340

12. Ahuja A, Ahuja NK. Clinical role-play in autonomous sensory meridian response (ASMR) videos: performance and placebo in the digital era. JAMA 2019; 321(14):1336-1337. doi:10.1001/jama.2019.2302

13. Barratt EL, Spence C, Davis NJ. Sensory determinants of the autonomous sensory meridian response (ASMR): understanding the triggers. PeerJ 2017 ;5:e3846. doi:10.7717/peerj.3846

14. Smith SD, Fredborg BK, Kornelsen J. A functional magnetic resonance imaging investigation of the autonomous sensory meridian response. PeerJ 2019; 7:e7122. doi:10.7717/peerj.7122

15. Smith SD, Katherine Fredborg B, Kornelsen J. An examination of the default mode network in individuals with autonomous sensory meridian response (ASMR). Soc Neurosci 2017; 12(4):361-365. doi:10.1080/17470919.2016.1188851
16. Smith SD, Fredborg BK, Kornelsen J. Atypical functional connectivity associated with autonomous sensory meridian response: an examination of five resting-state networks. Brain Connect 2019; 9(6):508-518. doi:10.1089/brain.2018.0618

17. Wang $X$, Yang $X$, Sun $Y$, Su $Y$. The influence of autonomous sensory meridian response on individual's executive function. Q J Exp Psychol (Hove) 2020; 73(10):1587-1595. doi:10.1177/1747021820922954

18. Beck AT, Steer RA, Brown GK. Manual for the Beck depression inventory-II. San Antonio, TX: Psychological Corporation; 1996.

19. Beck AT, Epstein N, Brown G, Steer RA. An inventory for measuring clinical anxiety: psychometric properties. J Consult Clin Psychol 1988; 56(6):893-897. doi:10.1037//0022-006x.56.6.893

20. Hofmann SG, Gómez AF. Mindfulness-based interventions for anxiety and depression. Psychiatr Clin North Am 2017; 40(4):739-749. doi:10.1016/j.psc.2017.08.008

21. Kabat-Zinn J, Lipworth L, Burney R. The clinical use of mindfulness meditation for the self-regulation of chronic pain. J Behav Med 1985; 8(2):163-190. doi:10.1007/BF00845519

22. Hilton L, Hempel S, Ewing BA, et al. Mindfulness meditation for chronic pain: systematic review and meta-analysis. Ann Behav Med 2017; 51(2):199-213. doi:10.1007/s12160-016-9844-2

23. Warr P, Bindl UK, Parker SK, Inceoglu I. Four-quadrant investigation of job-related affects and behaviours. Eur J Work Organ Psychol 2014; 23(3):342-363. doi:10.1080/1359432X.2012.744449

24. Gatti E, Calzolari E, Maggioni E, Obrist M. Emotional ratings and skin conductance response to visual, auditory and haptic stimuli. Sci Data 2018; 5:180120. doi:10.1038/sdata.2018.120

25. Poerio GL, Blakey E, Hostler TJ, Veltri T. More than a feeling: autonomous sensory meridian response (ASMR) is characterized by reliable changes in affect and physiology. PLoS One 2018; 13(6):e0196645. doi:10.1371/journal.pone.0196645

26. Cash DK, Heisick LL, Papesh MH. Expectancy effects in the autonomous sensory meridian response. PeerJ 2018; 6:e5229. doi:10.7717/peerj.5229

27. Hostler TJ, Poerio GL, Blakey E. Still more than a feeling: commentary on Cash et al, "Expectancy effects in the autonomous sensory meridian response" and recommendations for measurement in future ASMR research. Multisens Res 2019; 32(6):521-531. doi:10.1163/22134808-20191366

28. Keizer A, Chang THR, O'Mahony CJ, Schaap NS, Stone KD. Individuals who experience autonomous sensory meridian response have higher levels of sensory suggestibility. Perception 2020; 49(1):113116. doi:10.1177/0301006619891913

29. De Pascalis V, Chiaradia C, Carotenuto E. The contribution of suggestibility and expectation to placebo analgesia phenomenon in an experimental setting. Pain 2002; 96(3):393-402. doi:10.1016/s0304-3959(01)00485-7

Address: Arya B. Mohabbat, MD, Division of General Internal Medicine, Mayo Clinic, 200 First Street SW, Rochester, MN 55905; mohabbat.arya@mayo.edu 